\title{
Towards Accurate and Efficient Reliability Modeling of Nanoelectronic Circuits
}

\author{
Erin Taylor, Jie Han, and José Fortes \\ Department of Computer and Electrical Engineering \\ University of Florida, Gainesville, Florida \\ \{ertaylor, jiehan, fortes\}@acis.ufl.edu
}

\begin{abstract}
The emergence of nanoelectronic devices which rely on fundamentally unreliable physics calls for reliability evaluation techniques and practical design-for-reliability solutions. This paper reviews a method that uses probabilistic gate models (PGMs) for reliability estimation and improves upon this method to enable the accurate evaluation of reliabilities of circuits. When applied to large, complex circuits, however, this and other accurate methods lead to long execution times. To simplify reliability analysis, this paper leverages the fact that many large circuits consist of common logic modules. The overall circuit reliability estimation can be made on the basis of accurate PGM-based reliabilities of individual modules. This technique significantly reduces the PGM method's complexity, making it suitable for practical design-for-reliability applications. Results from the use of this technique on benchmark circuits indicate that the estimates produced correctly identify the most vulnerable paths through a circuit.
\end{abstract}

Keywords-Reliability, fault-tolerance, Nanoelectronics.

\section{INTRODUCTION}

Extensive efforts are being made to develop nanoelectronic devices implemented in nanoscale CMOS and in novel nanotechnologies such as quantum-dot cellular automata (QCA), single-electron tunneling devices (SET), carbon nanotubes, and molecular electronics. Fundamentally, any such nanoscale device will not be able to function as reliably as a comparable microscale device. This is due to device behavior dependencies on variations in size, temperature, power and quantum phenomena at the nanoscale.

At the nanoscale, precise control over the fabrication of devices will be extremely difficult, and this will lead to high defect rates and process variability, both of which will have a negative impact on the correct functioning and reliability of devices. These devices will also be more susceptible to soft errors due to their smaller geometries and reduced noise margins. At the same time, lower operating voltages and reduced power will increase device error probability due to thermal fluctuations. In the case of non-CMOS nanoelectronics, device reliability will be affected by the nondeterministic behavior and randomness inherent to selfassembly processes or sensitivity to background charges. Thus, nanoscale computing will have to deal with probabilistic and unreliable behavior of future nano-devices.

This concern for the reliability of nanoelectronics has motivated efforts to develop reliability evaluation techniques based on the probabilistic nature of future nanoscale computation $[1,2,3]$. This paper contributes to this work by developing a method for accurately and efficiently evaluating reliability at the logic-gate level. The probabilistic nature of this method makes it applicable to the analysis of logic circuits implemented with any nanotechnology. This approach is based on an approximate method summarized in section II. The accurate method is described in section III, and in section IV we introduce techniques to simplify the evaluation of reliability for use in practical circuit design.

\section{AN APPRoXimate ReLiAbILITy Model}

As discussed in [4], probabilistic gate models (PGMs) are equations that describe the behavior of unreliable logic gates. These models are constructed by assuming that a given logic gate has a probability of failure $\varepsilon$ and that failures occur according to a chosen fault model (e.g. von Neumann and stuck-at faults). Based on the chosen fault model, an equation is constructed to relate the gate's input values and $\varepsilon$ to the probability that the gate's output is ' 1 '. For example, consider a 2-input NAND gate where $X$ and $Y$ each denote the probability of an input being ' 1 '. Given that the gate is not faulty, the probability that the output is ' 1 ' equals $1-X Y$ and the probability the output is ' 0 ' equals $X Y$. Therefore, we can construct the following PGM for a 2-input NAND gate under the von Neumann fault model where a gate inverts its correct output value with probability $\varepsilon \leq 0.5: Z=(X Y) \varepsilon+(1-X Y)(1-\varepsilon)$. This model assumes that the inputs to a gate are independent.

One advantage of PGMs is their extensibility to any arbitrary gate and fault model. For example, PGMs for stuckat fault models are constructed in [5], and because they are not technology dependent, PGMs can also be used to model a number of nanoelectronic devices. QCA, for instance, implements logic gates using a number of interacting cells. Each QCA gate could be described by a PGM with the value $\varepsilon$ reflecting the error probability due to cell misalignment, background noise, or cell omission [6].

The use of PGMs to estimate circuit reliability, as introduced in [4], can be summarized as follows. In any PGM, both the input variables and the output variable represent the probability that a given signal is ' 1 '. Because of this, the output of one PGM can be used directly as the input to another PGM. Thus, for a given circuit, the PGM equation for each gate is applied in a topological order starting from primary inputs and proceeding to the circuit outputs. The final PGM equations give the probability that the circuit's outputs are ' 1 ', denoted $Z_{\varepsilon}$. Assuming no faults (i.e., $\varepsilon=0$ ), the PGM equations also provide the perfect circuit output, $Z_{\mathrm{p}}$. Circuit reliability is thus given by $\mathrm{R}=\mathrm{P}\left(\mathrm{Z}_{\varepsilon}=1\right) \mathrm{P}\left(\mathrm{Z}_{\mathrm{p}}=1\right)+\left(1-\mathrm{P}\left(\mathrm{Z}_{\varepsilon}=1\right)\right)\left(1-\mathrm{P}\left(\mathrm{Z}_{\mathrm{p}}=1\right)\right)$, which only requires knowledge of the probability that a circuit's outputs are '1'. For circuits with $n$ inputs, we consider 
all $2^{\mathrm{n}}$ input combinations to be equally probable, but in practice these input combinations can be weighted to reflect the actual distribution of inputs to a circuit.

This procedure can be extended to any circuit that does not contain cycles or feedback loops, since each PGM equation assumes independent inputs. In a circuit that contains fanouts, two or more of its signals will not be independent, resulting in inaccurate output probabilities. These unaccounted dependencies result in a circuit output probability that is an approximation of its actual value.

\section{AN ACCURATE REliability Model}

One advantage of the approximate PGM method is speed. However, accuracy cannot be guaranteed for a circuit with signal dependencies since the approximate method does not account for them. This has led us to consider a new technique that can accurately calculate the reliability of a circuit. In order to do so, we must account for signal dependencies, but maintaining this information is costly in terms of both speed and space. The method used in [3], for example, keeps track of the dependencies between all signals, whether they are necessary or not. So while it is accurate, its performance is not optimized.

We propose a method that keeps track of only those dependencies needed to calculate the accurate output reliability. Since the only sources of dependencies in a circuit without feedback are fanouts, our method relies on correctly modeling the signal dependencies introduced through these fanouts. In the simplest case, none of a fanout's branches reconverge along a path to a single output. This is called a nonconvergent fanout and the signal dependencies it introduces can be ignored when considering individual outputs. If there is a fanout with branches that reconverge along some paths to a single output (e.g. Fig. 1), it is a reconvergent fanout and we introduce the following two properties to remove the statistical dependence among fanout signals while maintaining an accurate circuit model.

For the first property, consider a fanout with an incoming signal $I$ and fanout branches $\mathrm{X}_{1}, \mathrm{X}_{2}, \ldots \mathrm{X}_{\mathrm{n}}$. We shall show that $X_{1}, X_{2}, \ldots X_{n}$ are statistically independent if $I=1$ or 0 . If $I=1$, then the joint probability $\mathrm{P}\left(\mathrm{X}_{1}=\mathrm{x}_{1}, \mathrm{X}_{2}=\mathrm{x}_{2}, \ldots \mathrm{X}_{\mathrm{n}}=\mathrm{x}_{\mathrm{n}}\right)=1$ only for $x_{1}=x_{2}=\ldots=x_{n}=1$. For any other values of $x_{1}, x_{2}, \ldots, x_{n}$, their joint probability is 0 . In this case we also have $\mathrm{P}(I)=\mathrm{P}\left(\mathrm{X}_{1}\right)=\mathrm{P}\left(\mathrm{X}_{2}\right)=\ldots=\mathrm{P}\left(\mathrm{X}_{\mathrm{n}}\right)$, so the product $\mathrm{P}\left(\mathrm{X}_{1}=\mathrm{x}_{1}\right) \mathrm{P}\left(\mathrm{X}_{2}=\mathrm{x}_{2}\right)$ $\ldots P\left(X_{n}=x_{n}\right)=1$ only for $x_{1}=x_{2}=\ldots=x_{n}=1$. In any other case, this product is 0 . Therefore, we have $\mathrm{P}\left(\mathrm{X}_{1}, \mathrm{X}_{2}, \ldots \mathrm{X}_{\mathrm{n}}\right)=$ $\mathrm{P}\left(\mathrm{X}_{1}\right) \mathrm{P}\left(\mathrm{X}_{2}\right) \ldots \mathrm{P}\left(\mathrm{X}_{\mathrm{n}}\right)$, which proves that for $I=1$, the fanout branches are mutually independent. The same can be shown for $I=0$ but not for $0<I<1$. We conclude that if the inputs to all fanouts in a circuit are 1 or 0 , then the signals are statistically independent.

To account for the signal probabilities at fanout inputs, we introduce the second property. If $\mathrm{I}_{\mathrm{i}}$ is the input to fanout $\mathrm{F}_{\mathrm{i}}$, then the probability that an output is ' 1 ' is given by $\mathrm{Z}=\mathrm{P}$ (output $\left.=1 \mid \mathrm{I}_{\mathrm{i}}=1\right) \mathrm{P}\left(\mathrm{I}_{\mathrm{i}}=1\right)+\mathrm{P}\left(\right.$ output $\left.=1 \mid \mathrm{I}_{\mathrm{i}}=0\right) \mathrm{P}\left(\mathrm{I}_{\mathrm{i}}=0\right)$. This simplifies to

$$
\mathrm{Z}=\mathrm{Z}_{\mathrm{i}}^{1} \mathrm{P}_{\mathrm{i}}+\mathrm{Z}_{\mathrm{i}}^{0}\left(1-\mathrm{P}_{\mathrm{i}}\right)
$$

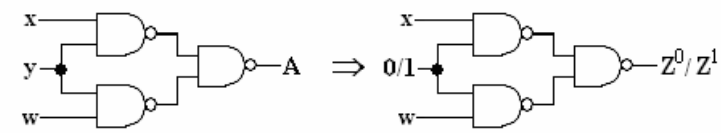

Figure 1. Illustration of reliability evaluation for a circuit with one reconvergent fanout. The fanout branches are set to the deterministic values ' 0 ' and then ' 1 ' in order to calculate the output values $Z^{0}$ and $Z^{1}$, respectively.

where $P_{i}$ is the probability that $I_{i}$ is ' 1 ' and $Z_{i}^{1}$ and $Z_{i}^{0}$ are the values of the output $Z$ when the input to fanout $F_{i}$ is set to ' 1 ', and ' 0 ', respectively. Using these two properties, we can accurately model any reconvergent fanout and hence, any signal dependencies in a circuit. To do this, the branches of the fanout are first set to deterministic 1's and 0's, and for each case, the outputs $Z^{1}$ and $Z^{0}$ are calculated. This process is shown in Fig. 1.

If all of the reconvergent fanouts in a circuit are mutually independent, they can be handled by considering the point of convergence (POC) of each individual fanout. We define the POC of fanout $F_{i}$ as the point where the branches of $F_{i}$ are combined so that the dependencies among the branches are resolved as a single output probability. A fanout is independent if there are no other fanouts or fanout branches in the path between its input and its POC. Fig. 2(a) shows a circuit with two independent fanouts. In this case, reliability is determined by considering each fanout individually and using the POC as the signal $\mathrm{Z}$ in equation (1).

We characterize dependencies of reconvergent fanouts as either parallel or nested depending on whether they are introduced through a fanout or a fanout branch. In the case of parallel fanouts, the dependency arises when one or more branches from the set of fanouts $\left\{\mathrm{F}_{\mathrm{k}}, \ldots \mathrm{F}_{\mathrm{k}+\mathrm{j}}\right\}$ are in the path between fanout $F_{i}$ and $F_{i}$ 's POC, where $i \neq k \neq \ldots \neq k+j$. It is only the branches from $\left\{\mathrm{F}_{\mathrm{k}}, \ldots \mathrm{F}_{\mathrm{k}+\mathrm{j}}\right\}$ that are in the path and not the fanouts' input. When $j=0$, illustrated in Fig.2(b), $F_{k}$ and $F_{i}$ are considered jointly as parallel fanouts. First the branches of $F_{k}$ and $F_{i}$ are both set to ' 0 ' and the signal probability at $F_{i}$ 's POC is evaluated. Then $F_{k}$ is set to ' 1 ' while $F_{i}$ remains ' 0 ' and the process is repeated until all $2^{2}$ different combinations of values at $\mathrm{F}_{\mathrm{i}}, \mathrm{F}_{\mathrm{k}}$ are evaluated. Equation (1) is modified as follows in order to calculate the final probability at the POC:

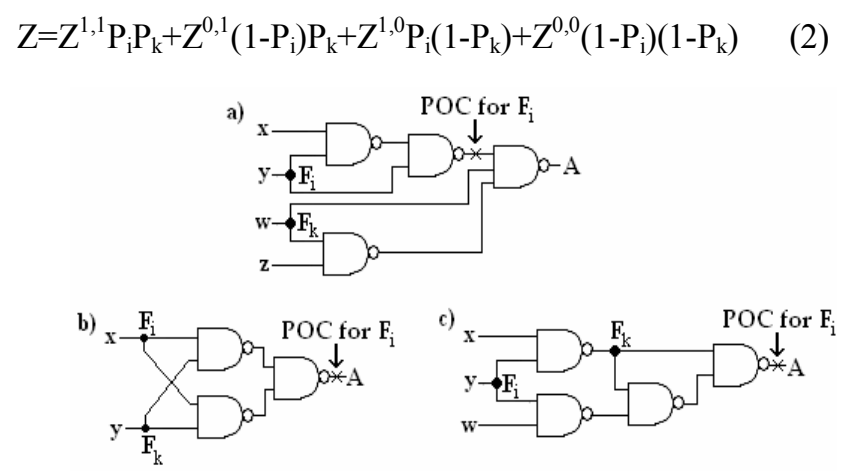

Figure 2. a) A circuit with a two independent fanouts, $F_{i}$ and $F_{k}$, b) a circuit with two parallel fanouts, $F_{i}$ and $F_{k}$, and c) a circuit with a nested fanout. In each circuit, $X$ marks the point of convergence of fanout $\mathrm{F}_{\mathrm{i}}$. 
where $\mathrm{P}_{\mathrm{i}}$ and $\mathrm{P}_{\mathrm{k}}$ are the probabilities that the input to fanout $i$ and fanout $k$ is ' 1 ', respectively. $Z^{\mathrm{m}, \mathrm{n}}, m, n \in\{0,1\}$, is the value at the POC, $Z$, when the branches of fanout $F_{i}$ are set to the value $m$ and the branches of $\mathrm{F}_{\mathrm{k}}$ are set to $n$. This process can be easily extended to the case when $\mathrm{j}>0$ and there will be $2^{\mathrm{j}+2}$ different settings for the branches of fanouts $F_{i}$ and $F_{k}, \ldots F_{k+j}$ since they are all considered jointly.

Nested dependencies occur when a set of fanouts, $\left\{\mathrm{F}_{\mathrm{k}}, \ldots \mathrm{F}_{\mathrm{k}+\mathrm{j}}\right\}$, are in the path between fanout $\mathrm{F}_{\mathrm{i}}$ and $\mathrm{F}_{\mathrm{i}}$ 's POC, where $\mathrm{i} \neq \mathrm{k} \neq \ldots \neq \mathrm{k}+\mathrm{j}$. When $\mathrm{j}=0$, we have the case illustrated in Fig. 2(c). We evaluate this scenario by first setting $F_{i}$ 's branches to ' 1 ' and calculating the probability that the input to fanout $F_{k}$ is ' 1 '. This probability will be conditioned on $F_{i}$ being ' 1 ', so we denote this as $\mathrm{F}_{\mathrm{k}}{ }^{1}$ where $\mathrm{F}_{\mathrm{k}}{ }^{1} \triangleq \mathrm{P}\left(\mathrm{F}_{\mathrm{k}}=1 \mid \mathrm{F}_{\mathrm{i}}=1\right)$. Then we set $F_{k}$ to ' 1 ' and find the signal probability at $F_{i}$ 's $P O C$, which we denote as $Z^{1,1}$. Next, $F_{k}$ is set to ' 0 ' and we calculate the POC value $Z^{1,0}$. We repeat this process for $F_{i}$ equal to ' 0 ' which yields the terms $F_{k}{ }^{0}$ where $\mathrm{F}_{\mathrm{k}}^{0} \triangleq \mathrm{P}\left(\mathrm{F}_{\mathrm{k}}=1 \mid \mathrm{F}_{\mathrm{i}}=0\right), \mathrm{Z}^{0,1}$ and $\mathrm{Z}^{0,0}$. Equation (1) is modified in the following way to calculate the final POC probability:

$\mathrm{Z}=\mathrm{Z}^{1,1} \mathrm{P}_{\mathrm{i}} \mathrm{F}_{\mathrm{k}}{ }^{1}+\mathrm{Z}^{1,0} \mathrm{P}_{\mathrm{i}}\left(1-\mathrm{F}_{\mathrm{k}}{ }^{1}\right)+\mathrm{Z}^{0,1}\left(1-\mathrm{P}_{\mathrm{i}}\right) \mathrm{F}_{\mathrm{k}}{ }^{0}+\mathrm{Z}^{0,0}\left(1-\mathrm{P}_{\mathrm{i}}\right)\left(1-\mathrm{F}_{\mathrm{k}}{ }^{0}\right)$

where $\mathrm{P}_{\mathrm{i}}$ is the probability that the input to fanout $i$ is ' 1 '. This process can be extended to the case when $\mathrm{j}>0$ by first considering $\mathrm{F}_{\mathrm{i}}$ and then continuing to each fanout in $\left\{\mathrm{F}_{\mathrm{k}}, \ldots, \mathrm{F}_{\mathrm{k}+\mathrm{j}}\right\}$ in a topological order from primary inputs to outputs. The final equation for $\mathrm{F}_{\mathrm{i}}$ 's $\mathrm{POC}$ will be a function of $\mathrm{Z}^{\mathrm{m}_{1}, \mathrm{~m}_{2}, \ldots, \mathrm{m}_{\mathrm{j}+2}}$, where $\left\{\mathrm{m}_{1}, \mathrm{~m}_{2}, \ldots, \mathrm{m}_{\mathrm{j}+2}\right\}$ takes on all $2^{\mathrm{j}+2}$ different combinations of binary values.

Using these concepts, any circuit can be accurately modeled for reliability. In the best case, when there are no reconvergent fanouts, this method will have the same time complexity as the approximate method which grows linearly with the number of gates. However, when there are $n$ dependent reconvergent fanouts, the worst-case time complexity of this method will grow with $2^{\mathrm{n}}$.

\section{SIMPLIFYING RELIABILITY ANALYSIS}

As noted in [7], many large circuits contain a limited number of simple logic components that are used repeatedly throughout a design. This is especially true for the ISCAS-85 benchmarks which frequently utilize functional modules such as those shown in Fig. 3. Normally, accurate evaluation of the reliability of such large circuits is time-intensive regardless of the method used as complexity grows with the number of gates and signal dependencies. The time required to program a large circuit model and then perform the evaluation can be prohibitive, especially when designing for reliability. However, by realizing the presence of common logic components used throughout a design, we can simplify the analysis of reliability for practical applications.

First, we note that in designing for reliability, the goal is usually to improve those points in a circuit that are most vulnerable to errors [8]. For this purpose, it is useful to know the relative reliability of a circuit's outputs and, in particular, which path to an output has the lowest reliability. This can be determined by modeling the circuit using our approximate or accurate PGM method, but for a large circuit, both processes can take significant time to program and run. If the circuit contains repetitive logic modules, however, we can greatly simplify the analysis. Assuming these modules are relatively small, we can construct equations using the accurate PGM method to describe the functions they perform as if they were single gates and then maintain a table of the resulting equations. The equation for the 2-input XOR component in Fig. 3(a), for example, is

$A=\varepsilon(3+7 x y-6 x-6 y)+\varepsilon^{2}(-5-18 x y+13 x+13 y)+\varepsilon^{3}(2+$

$20 x y-12 x-12 y)+\varepsilon^{4}(4 y+4 x-8 x y)-y x+x+y$

With these equations, we can quickly obtain a reliability estimate for an output $X$ by determining the logic modules that lie along the path from the primary inputs to $X$. The equations for these modules are applied in a topological order without regard for signal dependencies between different modules. The output of the last module is used to estimate the reliability of $X$ as well as the reliability of the circuit path from the primary inputs to $X$. Using these estimates for the reliability, we can find the most vulnerable outputs and paths in a circuit and apply fault tolerant techniques to improve reliability. For circuits that contain large modules, we can apply this method recursively by first decomposing the large modules into smaller components which themselves may be composed of even smaller blocks of logic. Thus, the hierarchical structure of a circuit can be exploited to efficiently apply this modularized method.

In most practical applications, the modules contained in a circuit are known a priori since design is done using libraries of common logic components and the focus is on the higher module level rather than the logic gate level. In cases when these modules are not known, the techniques used in [7] can be employed in order to establish the modules contained in a circuit. These include searching a circuit description for specific blocks of code that match those of known modules or using design software to convert the description into a graphical schematic which can then be visually inspected.

To illustrate this technique, we considered the 74283 circuit, a 4-bit fast-adder with 9 inputs, 5 outputs and 36 gates. For our evaluations, we set $\varepsilon=0.05$ and assumed that all $2^{9}$ input combinations are equally probable. All computations are done using a conventional desktop machine with a $2.0 \mathrm{GHz}$ Pentium 4 processor. Applying the accurate method requires only $60 \mathrm{~s}$ of runtime, but due to the large number of reconvergent fanouts, programming this model takes significantly longer. Instead, we note that this circuit is composed of two common logic components, M1 and a carrylookahead generator (CLA), shown in Fig 3(b,c). The CLA

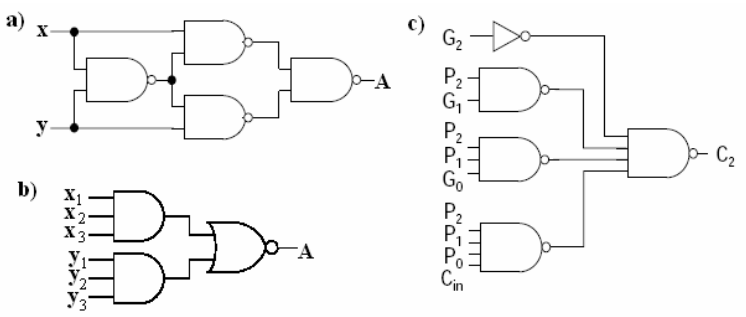

Figure 3. Common logic modules found in circuits include a) 2-input $X O R$, b) 6-input M1, and c) carry generator for third bit of CLA, from [7]. 
itself is a large module, so we break this down further into four smaller functional blocks, CLA1-CLA4. The circuit output $\mathrm{C} 4$, for example, is obtained by calculating the output of the following sequence of module equations: $(\mathrm{A}, \mathrm{B}) \rightarrow \mathrm{M} 1$ $\rightarrow$ CLA1 $\rightarrow(\mathrm{C} 4)$, where A and B are the circuit inputs. In this case, CLA1 depends on the outputs of four different M1 modules, each of which depend on two different circuit inputs. So we first use the equation for the M1 module to calculate the outputs of the four M1 modules and then use these values as the input to the CLA1 equation which outputs the probability of $\mathrm{C} 4$.

The output reliabilities obtained from both the accurate algorithm and the modularized method are shown in Table I for two benchmark circuits, the 74283 and the 74181, which is a 4-bit ALU containing 61 gates. The modularized method correctly predicted the least reliable output for both circuits. The estimates for the smaller 74283 circuit had a maximum error of just $3 \%$, while a larger discrepancy resulted in estimating the reliability of the larger 74181 circuit. However, runtime was reduced by $97.5 \%$ for the 74283 and by $99.9 \%$ for the 74181 circuit. This speedup is due to the fact that the signal dependencies among modules are not considered, as well as the fact that the module equations do not have to be reformulated each time they appear in the circuit. By having a table that stores the equations for common circuit components, we can significantly reduce not only runtime, but programming effort as well.

\section{CONCLUSION}

As CMOS approaches its scaling limits and new nanoelectronics emerge, reliability will be a major concern. To meet the need for reliability evaluation techniques, we have introduced the PGM method for modeling circuit reliability and have shown that it can be used to obtain accurate reliability measures. The model's flexibility allows it to be extended to numerous device technologies from nanoscale CMOS to emerging nanodevices.

TABLE I. SimULATION RESUlTS

\begin{tabular}{|c|c|c|c|c|c|}
\hline \multicolumn{3}{|c|}{ Reliability of 74283} & \multicolumn{3}{c|}{ Reliability of 74181 } \\
\hline Output & Accurate & Estimates & Output & Accurate & Estimates \\
\hline C4 & 0.770 & 0.780 & G & 0.827 & 0.838 \\
\hline S0 & 0.720 & 0.709 & A $=$ B & 0.762 & 0.815 \\
\hline S1 & 0.735 & 0.737 & F0 & 0.829 & 0.719 \\
\hline S2 & 0.749 & 0.740 & F1 & 0.799 & 0.653 \\
\hline S3 & 0.812 & 0.835 & F2 & 0.656 & 0.642 \\
\hline Time & $60 \mathrm{~s}$ & $1.6 \mathrm{~s}$ & Time & $10 \mathrm{hrs}$ & $35 \mathrm{~s}$ \\
\hline
\end{tabular}

Considering the fact that most large circuits are composed of a limited number of sub-modules, a hierarchical, modularized method is proposed for practical design applications. Large modules can be decomposed into their smaller components and then probabilistic equations that describe the functions of these small modules can easily be determined. Circuit reliability estimates are obtained by recursively applying the PGM method to this hierarchy of modules. Our results indicate that this method produces reasonably accurate reliabilities with minimal programming effort and processing time. Therefore, this technique is particularly useful in design-for-reliability applications where it is important to identify critical paths in a circuit. In future work, we envision incorporating other methods of reliability evaluation [1-3] with this hierarchical, modularized approach in order to improve accuracy.

\section{ACKNOWLEDGEMENT}

This work is supported in part by the NASA award no. NCC 2-1363.

\section{REFERENCES}

[1] R.I. Bahar, J. Chen and J. Mundy, "A probabilistic-based design for nanoscale computation," in Shukla and Bahar, eds. Nano, Quantum and Molecular Computing, 2004, pp. 133-156.

[2] T. Rejimon and S. Bhanja, "Scalable Probabilistic Computing Models using Bayesian Networks." IEEE Intl. Midwest Symp. on Circuits and Systems, 2005.

[3] S.Krishnaswamy, G.Viamontes, I.Markov, and J.Hayes, "Accurate Reliability Evaluation and Enhancement via Probabilisitic Transfer Matrices." Proc. Design, Automation and Test in Europe Conf., 2005.

[4] J. Han, E. Taylor, J. Gao, and J. Fortes. "Reliability Modeling of Nanoelectronic Circuits." Proc. of IEEE Nano., 2005.

[5] J. Han, E. Taylor, J. Gao and J. Fortes, "Faults, Error Bounds and Reliability of Nanoelectronic Circuits," Proc. IEEE ASAP, 2005.

[6] M. Tahoori, M. Momenzadeh, J. Huang, and F. Lombardi. "Defects and Faults in Quantum Cellular Automata at Nano Scale." Proc. of IEEE VLSI Test Symp., 2004.

[7] M.C. Hansen, H. Yalcin, and J. P. Hayes. "Unveiling the ISCAS85 Benchmarks." IEEE Design and Test of Computers, 1999.

[8] K. Mohanram and N. A. Touba. "Cost-Effective Approach for Reducing Soft Error Failure Rate in Logic Circuits." Proc. Intl. Test Conference, 2003. 